INGENIERÍA AMBIENTAL

\title{
Sistema de solución creativa para problemas recurrentes - Itacone
}

ENVIRONMENTAL ENGINEERING

\section{Itacone - A creative solution system to recurrent problems}

\author{
Jaime A. Romero-Infante*§, Rafael A. Moré-Jaramillo* \\ * Universidad El Bosque. Programa Ingeniería Ambiental. Programa Administración de Empresas \\ §romerojaimea@unbosque.edu.co,morerafael@unbosque.edu.co
}

(Recibido: Octubre 13 de 2011- Aceptado: Mayo 16 de 2013)

\begin{abstract}
RESUMEN
Itacone es un sistema creado para resolver problemas recurrentes que requieren el uso de técnicas creativas, teniendo en cuenta las particularidades culturales y socioeconómicas de la comunidad latinoamericana, preparando la mente del consultor que lo aplica, para conocer lo que piensan las partes involucradas en resolver el problema, cambiar la forma de ver el mismo, analizar objetivamente ideas de solución y generar una estrategia que incluya las soluciones que mejor se adaptan a resolver ese problema integralmente, teniendo en cuenta las consecuencias de esa solución en el entorno natural y social. El sistema se basa en propuestas de investigadores canadienses (Briand, A. 2004) y latinoamericanos (los autores) para definir el problema a estudiar de manera objetiva, en investigadores europeos para romper paradigmas con relación a soluciones que han fracasado con anterioridad y utiliza el concepto de la logística en reversa (o logística inversa entendida como las actividades desarrolladas para revisar las consecuencias de un producto con el propósito de maximizar su valor, Angulo 2000), verificando la aplicabilidad de la solución diseñada con los dueños del problema antes de entregarla definitivamente, y eventualmente conocer las consecuencias de aplicar la solución estratégica para ajustarla y maximizar sus posibilidades de éxito.
\end{abstract}

Palabras claves: Creatividad, organizaciones, problemas, soluciones, sustentabilidad.

\begin{abstract}
Itacone is a system created to solve recurring problems that require the use of creative techniques, taking into account the cultural and socio-economic of the Latino community. The system prepares the mind Itacone consultant applying the method to know what they think the other people involved in it, change the way you see the problem, solution ideas objectively analyze and generate a strategy that includes the solutions that best adapted to solve the problem comprehensively. The proposed method is based on Canadian and American researchers to define the problem to be studied objectively in European researchers to break paradigms with regard to solutions that have failed before and uses the concept of reverse logistics in order to verify the applicability of the solution is being designed with the owners of the problem before final delivery and eventually understand the consequences of applying the proposed strategic solution to fit so as to maximize their chances of success.
\end{abstract}

Keywords: Creativity, organizations, problem, solution, sustainability. 


\section{Introducción}

ITACONE es el nombre asignado por los autores para el sistema de solución creativa a problemas recurrentes y que hace referencia a los vocablos muiscas ITA que significa "mano" y CONE que significa "apoyo, amigo", esto es una herramienta amiga para resolver problemas; la anterior etimología tiene como referencia el autor Miguel Triana en su libro La Civilización Chibcha, publicada por la Escuela Tipográfica Salesiana en 1922 y reimpresa por la Empresa Editorial Carvajal en 1972.

El desarrollo actual de la región latinoamericana hace necesaria la adopción de nuevas alternativas de solución a las problemáticas y prácticas tradicionales, para que su crecimiento sea sustentable y para que aquellos problemas que no se han podido resolver por más que quienes tengan los problemas hayan tratado de resolverlos, encuentren y apliquen definitivamente su solución de manera satisfactoria.

Las situaciones de pobreza, hambruna, violencia, desigualdad, deterioro ambiental, entre otros que agobian la región, muestran que los problemas recurrentes no se están solucionando desde su causa incrementando en la mayoría de los casos sus consecuencias negativas. El sistema de solución creativa para problemas recurrentes ITACONE, surge a partir de la necesidad de dar soluciones viables, aplicables, sustentables y consecuentes a diferentes situaciones insatisfactorias que no se logran convertir en situaciones ideales. De esta forma, el desarrollo de la ciencia genera métodos que, apartándose de la realidad en la que surge el problema, producen las mejores soluciones que al implementarse logren la satisfacción de las necesidades de las empresas y comunidades, generando un modelo de solución creativa. Albert Einstein dijo una vez: "se requieren nuevas formas de pensar para resolver los problemas creados por las viejas formas de pensar" Gary, (2007).

Para claridad y comprensión del lector, a continuación se presentan los actores que se consideran involucrados en la solución creativa a un problema recurrente: Consultor: es la persona que externamente va a generar una estrategia de solución al problema recurrente para el dueño del problema. Dueño del problema: es el responsable de tener resuelto el problema y quien se lo plantea o quien tiene la responsabilidad de planteárselo; hasta que exista alguien responsable y consciente de resolver el problema, se podrá comenzar a resolverlo porque de lo contrario a nadie le interesará su solución o no habrá alguien interesado en ello (Briand, 2004). Contrastados: son las personas que están involucradas en el problema y que pueden tener percepciones diferentes del mismo, complementarias a aquellas que tiene el dueño del problema. Afectados del problema: son las personas que sufren las consecuencias de la existencia del problema. Y para terminar, a continuación se precisan los conceptos de Analogía, Sustentabilidad, Recurrencia y Solución creativa, dada la multiplicidad de acepciones que estos términos tienen y su importancia en las definiciones de los conceptos del Sistema ITACONE que se propone en el artículo.

Analogía: es el método que se toma para entender mejor el problema, cuyas características se analizan comparando los atributos del problema con los atributos de un sistema distinto, para describir el problema de una forma diferente de cómo se acostumbra a ver. Este método se basa en la propuesta de Gordon (llamada la sinéctica) en el que un problema se puede resolver comparándolo con otra cosa, "se trata de poner en paralelo mediante este mecanismo unos hechos, unos conocimientos o unas disciplinas distintas" (Almirón, 2010). Sustentabilidad: Consiste en aquella condición de quien actúa dándole buen uso a los recursos que utiliza, garantizando que siga disponiendo de ellos. Se refiere al equilibrio económico, ecológico y social que debe tener el ejercicio productivo para que pueda seguir existiendo en el futuro (Angel, 1995). Recurrencia: se entiende en este artículo como una situación que se presenta varias veces o de manera repetitiva en un proceso. Solución 
creativa: se considera una solución como el conjunto de actividades que se desarrollan para convertir la situación insatisfactoria en la situación ideal de una realidad problema, mientras que la solución creativa se entiende como aquella solución que aborda la problemática desde una perspectiva diferente de aquella en la que se genera el problema. Esa perspectiva se circunscribe a lo que considera la organización que posee el problema.

Este método resulta particularmente útil para los casos de las problemáticas ambientales empresariales y de creación sustentable de valor (aunque no sea el único caso en que se puede usar) que no han sido resueltas porque las personas quieren resolverlas de la misma manera como las crean; el método se constituye en un procedimiento general verificado en sus consecuencias y aplicabilidad por quien lo usa, para cambiar de paradigmas y producir de forma diferente a como lo venía haciendo para convertirse en producción sustentable. Por último, en el presente artículo ha sido de gran importancia el aporte que hizo la estudiante Andrea Gutiérrez del programa de Ingeniería Ambiental de la Universidad El Bosque y la Doctora Mónica Sofía Rico de la Pontificia Lateranensis de Roma por su corrección de estilo.

Generalmente los problemas en comunidades y en empresas se tratan de resolver por los mismos dueños del problema o por consultores que son contratados por los dueños del problema pensando de la misma manera como los crean. Se toma en este documento un problema recurrente como aquel que se ha tratado de resolver en anteriores oportunidades y que al aplicar la solución el problema persiste en la comunidad o en la empresa que lo sufre.

\subsection{Marco referencial}

\subsubsection{Creatividad en la ciencia}

"La verdad es que aquellos que nunca se han ocupado de cuestiones científicas conocen sólo una pequeña fracción de la poesía que les rodea. H. SPENCER
"Si bien seguir los pasos del modelo racional de la toma de decisiones con frecuencia mejora éstas, quien las tome también necesita creatividad, es decir, la aptitud de producir ideas novedosas y útiles" (Amabile, 1988). Según Robbins \& Judge (2009), la creatividad en la toma de decisiones es importante porque permite que quien las tome evalué y entienda por completo el problema, inclusive porque ve dificultades que otros no son capaces de detectar. Según Feist \& Barron (2003), la mayoría de personas inteligentes son creativas; Woodman, et al (1993), afirman que las características asociadas con la gente creativa son la independencia, confianza en sí mismo, propensión al riesgo, locus interno del control, tolerancia a la ambigüedad, poca necesidad de estructura y perseverancia ante la frustración. La información teórica sobre la creatividad es amplia y más los actores que se enfocan en el camino hacia la solución de problemas. Uno de ellos es William J.J. Gordon el cual propone un método que descubre los mecanismos psicológicos básicos de la creatividad el cual llama sinéctica (unión de elementos distintos y aparentemente irrelevantes). La sinéctica define al proceso creativo como la actividad mental desarrollada en aquellas situaciones donde se plantean y se resuelven problemas, con el resultado de invenciones artísticas o técnicas, Gordon, (2010)

La creatividad es innata en el ser humano, naturalmente su capacidad creativa hace que cada individuo desarrolle acciones diferentes a situaciones comunes. Sin embargo, una vez el individuo se sumerge en el sistema social, su creatividad se ve nublada por las ideas concebidas por otros, Esquivias, (2004). La creatividad es algo inherentea los sujetos. Cuando niños explorábamos creativamente el mundo, ahora adultos es una capacidad que por momentos desconocemos, una capacidad que puede entrenarse. Según (Fabris et. al, 2008), este entrenamiento consiste fundamentalmente en un "desaprender" conductas que limitan la creatividad. Aprender a eludir los "bloqueos", desbloquearse. Según Fidel Moccio, (1991), la creatividad es producto de un estado, al que denomina estado creativo (Estado creativo 
significa síntesis en varios sentidos: síntesis de mente y cuerpo, de conciencia e inconsciencia, sentimiento y razón, o sea de partes de una totalidad que están disociadas, funcionalmente separadas). Este es un estado que suele presentarse en ocasiones en la vida, particularmente cuando se consideran algunos problemas de manera inédita para nosotros mismos. De esta forma, la creatividad humana se vuelve una oportunidad para los que quieran salirse del sistema y generar nuevas ideas y ciencia, agregando valor y soluciones viables en y para la sociedad. Es así, como el carácter intangible de la creatividad se vuelve tangible en la medida que el sistema acepte $\mathrm{y}$ adopte satisfactoriamente las nuevas ideas, las cuales como soluciones responden en la mayoría de los casos a problemas tales como, pobreza, deterioro ambiental, maltrato intrafamiliar, mortalidad infantil, entre otros.

Según Silva García, (2010), “El hombre, como ser social, modifica su medio natural, construye obras arquitectónicas y urbanísticas, moldea objetos, en definitiva, crea, diseña y produce bienes materiales concretos y tangibles. Estas expresiones adquieren un sentido completo sólo cuando puede revelarse, más allá del objeto en sí, su valor subyacente. El hombre construye también otro tipo de manifestaciones a las que les otorga una significación particular, las que se expresan en una forma intangible e inmaterial. Son los bienes que dan cuenta de una identidad enraizada en el pasado, con memoria en el presente, reinterpretadas por las sucesivas generaciones, que tienen que ver con saberes cotidianos, prácticas familiares, entramados sociales $y$ convivencias diarias. Estos bienes hablan, por ejemplo, de la singularidad de ciertos oficios, músicas, bailes, creencias, lugares, comidas, expresiones artísticas, rituales o recorridos de "escaso valor físico pero con una fuerte carga simbólica".

A esta suma de patrimonios diversos denominamos Patrimonio Intangible. Todas sus manifestaciones son complejas, dinámicas y por lo tanto modificables y mantienen una interdependencia mutua. Tanto el Patrimonio Tangible, como el
Intangible componen el Patrimonio Cultural de cada grupo social. Se construyen históricamente, como resultado de las interacciones sociales, $\mathrm{y}$ otorgan especial sentido de pertenencia e identidad a la sociedad que los originó. Mantienen entre sí una relación dialéctica ya que lo "tangible logra mostrarse en toda su riqueza en tanto deja al descubierto su alma intangible. Por su parte lo intangible se vuelve más cercano y aprehensible en tanto se expresa a través del soporte de lo material'. Según García (2010), el patrimonio intangible impregna cada aspecto de la vida del individuo y está presente en todos los bienes que componen el patrimonio cultural: Monumentos, objetos, paisajes y sitios. Todos estos elementos, productos de la creatividad humana, y por lo tanto hechos culturales, se heredan, se transmiten, modifican y optimizan de individuo a individuo y de generación a generación.

Los patrimonios culturales que se arraigan en los individuos latinoamericanos, orientan la creatividad a concebir la búsqueda de soluciones de necesidades insatisfechas hacia soluciones ancestrales, raizales, tecnológicas y sustentables que las satisfagan y obedezcan a las leyes naturales y sociales. Las antiguas civilizaciones americanas Maya, Azteca, Chibcha e Inca, heredan una creatividad diversa, proveniente de lo empírico y de las necesidades históricas de nuestra civilización, que desarrollan nuevas formas de pensamiento, cultura de vida y ciencia. Es así, como la creatividad latinoamericana, heredada y/o construida, lleva consigo el patrimonio cultural que nos hace generar ideas sustentables que buscan el equilibrio social, ecológico y económico. El modelo de los cuatro componentes de la creatividad, es un modelo científico que explica como funciona la creatividad en el individuo. Según Amabile (2012), puesto que la mayoría de las personas tiene la capacidad de ser al menos algo creativas, los individuos y organizaciones podrían implementar el modelo de los cuatro componentes de la creatividad para estimularla en el individuo.

Con base en los resultados de muchas investigaciones, este modelo propone que la 
creatividad individual en esencia requiere de experticia, aptitud para el pensamiento creativo, trabajo en ambiente que promueva la creatividad y motivación intrínseca para la terea. Los estudios confirman que entre más elevado sea el nivel que alcancen estos cuatro componentes, mayor será la capacidad creativida del individuo y de la organización. Según Robbins \& Judge (2009), la experiencia es el fundamento de todo el trabajo creativo. El potencial para la creatividad mejora cuando los individuos tienen aptitudes, conocimiento, eficiencia y experiencias similares en su campo de trabajo. De igual forma, las aptitudes para el pensamiento creativo, agrupa características personales asociadas con la creatividad, aptitud para utilizar analogías y el talento para ver situaciones que han sido familiares a la luz de un punto de vista diferente. Según Gordon (1961), algunas personas han desarrollado aptitudes creativas porque son capaces de mirar los problemas de una manera nueva. Hacen que lo extraño resulte familiar y lo que es familiar parezca extraño. Finalmente, la motivación intrínseca para la tarea, consiste en el deseo de trabajar en algo porque en lo personal resulta interesante, absorbente, emocionante, satisfactorio o desafiante. Este componente de motivación es lo que convierte la creatividad potencial en ideas creativas reales.

\subsubsection{Metodo científico}

"En ocasiones, el replanteamiento de un problema es más decisivo que el hallazgo de la solución, que puede ser un puro asunto de habilidad matemática o experimental. La capacidad de suscitar nuevas cuestiones, nuevas posibilidades de mirar viejos problemas requiere una imaginación creativa y determina los avances cientificos auténticos. "ALBERT EINSTEIN

Toda investigación o proceso lógico con validez universal para generar algún tipo de conocimiento requiere del método científico, con el cual se obtiene rigor y se da explicación o solución a problemas o se predicen fenómenos en un determinado tiempo y espacio. El proceso de investigación científica cuenta con diferentes enfoques, a saber: cualitativo, cuantitativo y mixto. Particularmente el método científico mixto se caracteriza por utilizar una técnica de análisis cualitativa y cuantitativa que involucra cada uno de los elementos característicos y fundamentales del proceso. Con base en esto, "el Sistema de Solución Creativa para Problemas Recurrentes ITACONE”, implementa el método científico mixto para su desarrollo, integrando estrategias propias del contexto cultural en la solución, validadas cuantitativa $\mathrm{y} / \mathrm{o}$ cualitativamente en la organización y puntualmente con los dueños del problema, como ocurre cuando se presenta inicialmente la definición del problema a investigar, identificación de la metodología a utilizar, la tabulación de las evidencias objetivas del proceso, su análisis y la síntesis respectiva en una estrategia integrada de solución. La idea principal del sistema de solución planteado es romper paradigmas, salir de la subjetividad, cambiar el contexto y abrir la mente a diferentes conceptos, percepciones y conocimientos asociados, para así realizar descubrimientos que se puedan ordenar holísticamente, promoviendo una estrategia de solución real, critica, integral y aplicable al problema en estudio.

\section{Metodología}

La metodología utilizada para desarrollar este estudio es la deductiva por cuanto a partir de la aplicación de los métodos creativos como el PEC (Briand, 2004) y su aplicación en casos de estudio, se deduce una nueva propuesta metodológica adaptada al contexto cultural latinoamericano para resolver los problemas recurrentes. Estos métodos son diseñados para resolver problemas en las organizaciones que han tratado de resolverlos pero que no lo han logrado hasta el momento. Para ello, se desarrolla el sistema Itacone a partir de la propuesta realizada por Andre Briand (2004), originalmente denominada el Proceso de Exanimación Creativa PEC, aplicándose en varios casos de estudio especialmente enfocados a problemas ambientales y de creación de valor sustentable, generando una adaptación 
cultural del PEC así como la incorporación de procedimientos apropiados para entender mejor el problema, estimular la generación de ideas de solución, clasificar las ideas de solución, y revisar que esa solución no genere impactos negativos y que sea aplicable a los ojos del dueño del problema. Al final, se muestra cómo la aplicación del sistema Itacone a problemas específicos produce soluciones creativas con mayor eficacia y adaptabilidad cultural y menor impacto en la naturaleza y la sociedad. O sea que esta investigación, usa el método deductivo para generar un nuevo método con adaptaciones bio psico sociales y culturales.

\section{Resultados y discusión}

El estudio parte de una propuesta sobre cómo se deben afrontar los problemas recurrentes de tal manera que se cambie el contexto en el cual se crea; para esto se utilizan conceptos de la dinámica organizacional (como el contraste de las opiniones para determinar la realidad de una situación) y de la psicología individual (como que la percepción de un individuo de la realidad está afectada por sus paradigmas) para complementar las opiniones que las personas tienen del problema y generar estrategias integradas de solución, para después probarlas mediante una especie de simulación $\mathrm{y}$ poder detectar posibles inconvenientes en su aplicación o consecuencias negativas en el entorno. Veamos cómo se plantea el método.

El modelo de utilidad organizacional para solución de problemas recurrentes itacone. El sistema de solución creativa para problemas recurrentes, ITACONE, está basado en el método científico mixto de investigación. Está compuesto por un conjunto de acciones que generan la solución estratégica a partir de una mejor definición del problema mismo que se pretende resolver y siguiendo una secuencia lógica de pasos que inducen creatividad. El sistema permite identificar amplia y profundamente el problema, rompiendo una serie de paradigmas de tal forma que se pueda salir del contexto que lo crea, para mirarlo sistémicamente desde su entorno. Para seguir detalladamente los pasos del modelo de utilidad se presenta la figura 1 , de la cual se da explicación detallada en seguida.

Para afrontar el planteamiento de un problema recurrente, lo primero que se debe realizar es observar detalladamente el problema determinado para identificar las situaciones insatisfactorias allí presentes, así como las situaciones ideales a las que se quisiera llegar, Briand, (2004).

Existen cinco aspectos básicos que se deben tener en cuenta para el planteamiento del problema: Identificar los Elementos del problema a resolver: la situación insatisfactoria actual y la situación ideal; describir el problema a resolver como la diferencia entre la situación insatisfactoria y la situación ideal; formular la hipótesis de las posibles causas que dan origen al problema (es una afirmación que se plantea como respuesta a un interrogante previamente establecido.

Por lo tanto, la hipótesis en la solución de un problema recurrente es una proposición que surge de la relación entre variables, que de forma tentativa enuncia los posibles medios a través de los cuales se da solución a dicho problema de investigación); contraste de la percepción del problema (la percepción del dueño del problema recurrente, generalmente tiene un agregado de subjetividad, por lo cual, se hace necesario implementar una serie de actividades para definir el problema critica y objetivamente.

Es necesario, indagar entre los actores involucrados en el problema y demás entes participativos, sobre la situación insatisfactoria, situación ideal y los medios a utilizar para convertir la situación insatisfactoria en ideal desde el punto de vista de cada uno, Briand, (2004). Teniendo en cuenta que la definición del problema es la diferencia que existe entre la situación ideal e insatisfactoria, se recomienda la tabla 1 para contrastar la situación actual e ideal en una organización. El contraste de las percepciones del problema es el punto clave para el buen desarrollo y funcionamiento del Sistema de Solución Creativa para Problemas Recurrentes, 


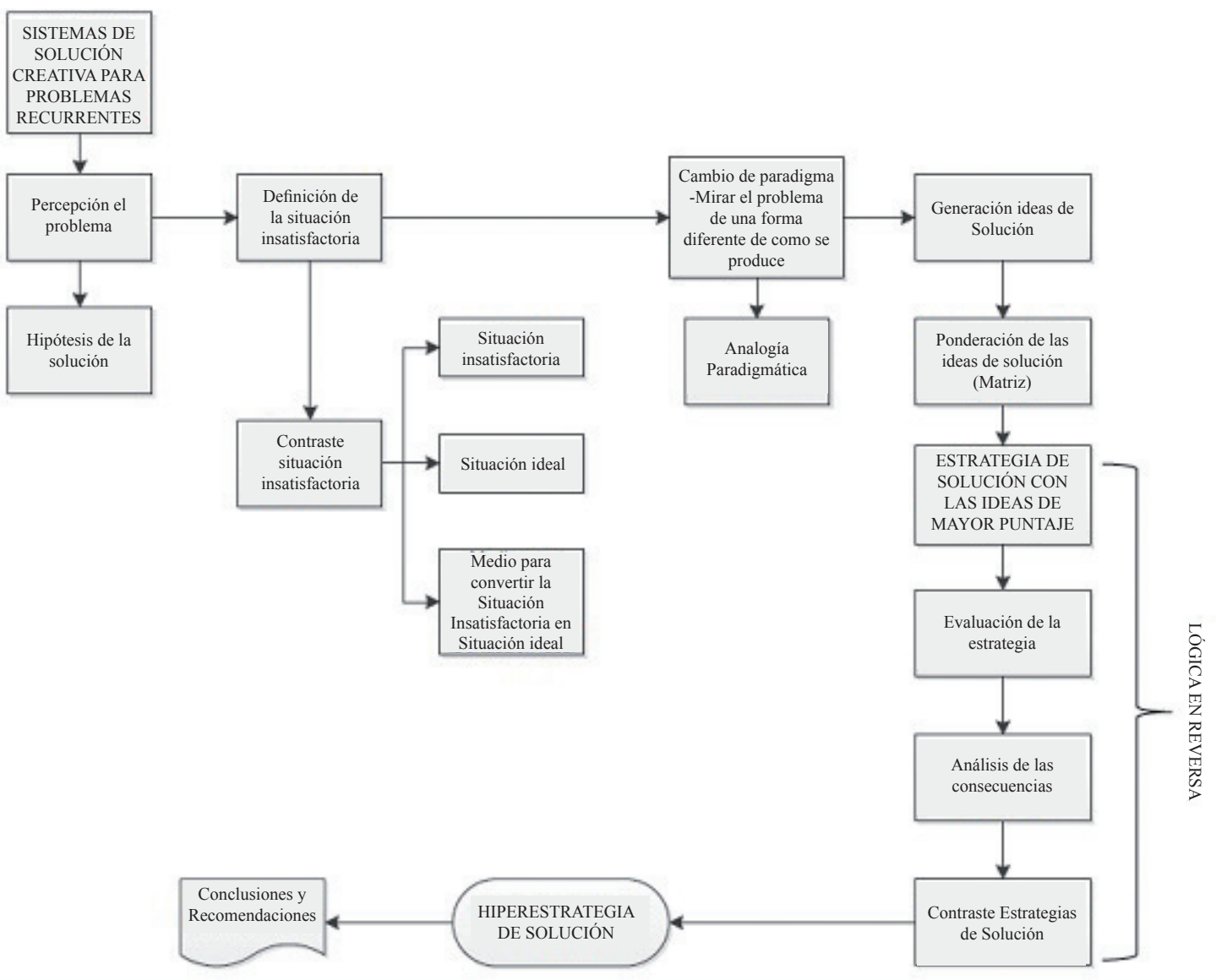

Figura 1. Lógica del sistema ITACONE.

ITACONE. Es importante resaltar que el modelo para contrastar la situación insatisfactoria se realiza de acuerdo a las preferencias y necesidades del consultor y; Definición del problema. Con la situación actual insatisfactoria y la situación ideal documentada entre los actores involucrados, el problema se define como la comparación entre las dos situaciones identificadas después del contraste. Esto es, el problema es una frase clara, concisa y contundente que compara la situación insatisfactoria con la situación ideal teniendo en cuenta las diferentes percepciones del problema.

Este marco es riguroso si se va a resolver un problema de investigación, si el problema es de gestión entre otros, estos marcos podrán ser tratados a discreción por un consultor encargado por el dueño del problema. Éste marco expone los tópicos principales y secundarios, las ideas centrales y auxiliares que se desarrollan en un informe o trabajo investigativo. Así mismo, se da un tratamiento a la historia del tema, es decir, investigaciones anteriores, posteriores, que marcan la evolución del tópico tratado. En el marco referencial se incluye un marco de conceptos y definiciones.

Para generar la solución estratégica al problema de manera corroborada, se ha escogido el término hipersolución como resultado de la comparación del problema mediante el uso de la analogía antiparadigmática, de una síntesis de las ideas de solución y del planteamiento de una estrategia de solución que luego se corrobora con la aplicación de la técnica analítica de lógica en reversa.

Analogía anti paradigmática. Una vez revisados y utilizados los métodos de creatividad, el que 
Tabla 1. Ejemplo para contrastar y ponderar las ideas de los individuos de la organización (Contraste de individuos de comunidad rural para solucionar sus problemas de manejo y disposición de residuos sólidos).

\begin{tabular}{|c|c|c|c|c|}
\hline No. & $\begin{array}{l}\text { Cargo y nombre de } \\
\text { la persona }\end{array}$ & Situación Actual & Situación Ideal & $\begin{array}{l}\text { Medio para resolver la } \\
\text { situación actual en Ideal }\end{array}$ \\
\hline 1 & $\begin{array}{l}\text { Líder comunidad / } \\
\text { Ramiro Huertas }\end{array}$ & $\begin{array}{l}\text { Los residuos que se producen en su mayoría son las cascaras } \\
\text { de los alimentos que salen de la huerta comunal, que al } \\
\text { acumularse producen malos olores en la zona. }\end{array}$ & $\begin{array}{l}\text { No ver residuos ni oler } \\
\text { malos olores dentro de } \\
\text { la comunidad. }\end{array}$ & $\begin{array}{l}\text { Acondicionar un lugar } \\
\text { cercano pero a la vez alejado } \\
\text { donde los olores no lleguen a } \\
\text { la comunidad. }\end{array}$ \\
\hline 2 & $\begin{array}{l}\text { Madre cabeza de } \\
\text { hogar / Patricia } \\
\text { Lozano }\end{array}$ & $\begin{array}{l}\text { En realidad en la casa se producen muy pocos residuos ya } \\
\text { que todos los que salen los utilizo en la huerta familiar que } \\
\text { se encuentra cercana a mi casa. Sin embargo, la cantidad de } \\
\text { residuos que salen de las demás casas están perjudicando a } \\
\text { todos los vecinos del sector. }\end{array}$ & $\begin{array}{l}\text { Que mis hijos puedan } \\
\text { crecer en un lugar libre } \\
\text { de olores y vectores. }\end{array}$ & $\begin{array}{l}\text { Que todas las casas cuenten } \\
\text { con huertas familiares } \\
\text { donde aprovechen todos los } \\
\text { residuos orgánicos que salen } \\
\text { de sus hogares. }\end{array}$ \\
\hline 3 & $\begin{array}{l}\text { Tendero / Augusto } \\
\text { Dueñas }\end{array}$ & $\begin{array}{l}\text { Al existir una huerta comunal y en algunas ocasiones huertas } \\
\text { familiares los residuos que se producen en su mayoría son de } \\
\text { comida y se descomponen rápido, sin embargo si hemos tenido } \\
\text { problemas con la acumulación de residuos de empaques de } \\
\text { otros alimentos que vendo acá en la tienda. }\end{array}$ & $\begin{array}{l}\text { Que el gobierno o } \\
\text { alcaldía nos solucione } \\
\text { el problema, para } \\
\text { que no se acumulen } \\
\text { los residuos no } \\
\text { biodegradables. }\end{array}$ & $\begin{array}{l}\text { Que venga un carro } \\
\text { recolector de basura } \\
\text { periódicamente } \\
\text { recoger esos residuos no } \\
\text { biodegradables y los lleve al } \\
\text { relleno más cercano. }\end{array}$ \\
\hline
\end{tabular}

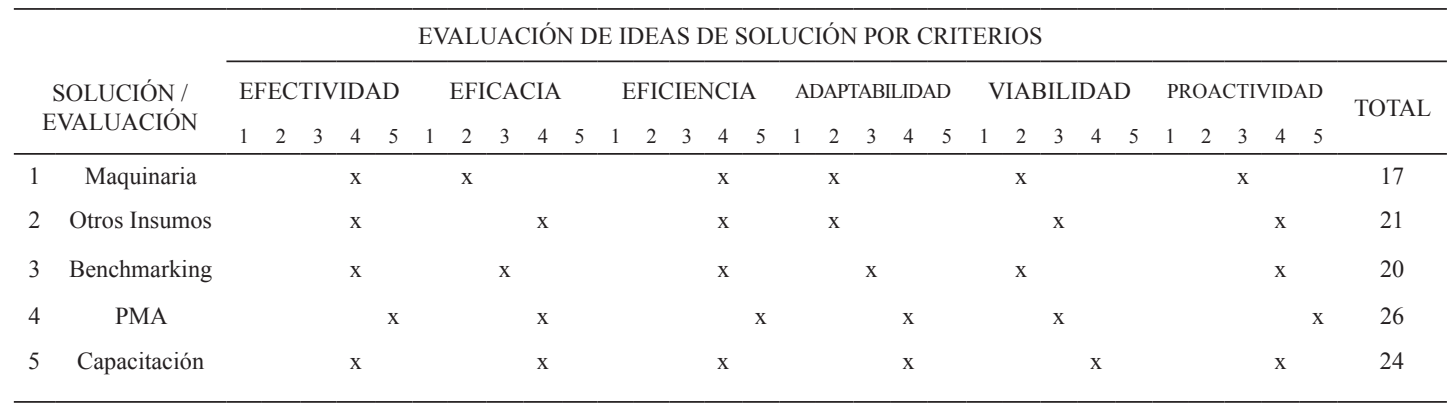

mejores resultados ha proporcionado y que más versátilmente se ha podido utilizar es el de la Analogía antiparadigmática. Para entender la realidad problémica, es conveniente analizarla mediante una analogía que rompa con los paradigmas que el dueño del problema tiene (por ejemplo, con sistemas hídricos, sistema humano, comportamiento de flora o fauna, entre otros). Es la analogía entre elementos que no mantengan relación alguna, aquella que más resulta útil porque ello le permite al consultor ver el problema de una manera diferente de cómo los demás la ven. Hacer la analogía implica comparar las partes del problema con las partes del sistema análogo de manera que el problema se comience a ver $\sin$ los paradigmas con los que se creo.

Según Fabris, et al (2008), la sinéctica de Gordon posibilita, a través del uso sistemático de la metáfora, el logro de un "estado creativo" en el que se hacen posibles las mencionadas "conexiones funcionantes y productivas", la "síntesis" y los "procesos integrativos", que son las condiciones para la emergencia de las imágenes e ideas creativas. La sinéctica propone con base en este estado creativo, el planteamiento de soluciones a problemas concretos. "Para ello se organiza una "excursión mental" cuyos pasos consisten en volver lo conocido extraño y lo extraño conocido. Según estos postulados, Gordon, (1992), existen 4 mecanismos para volver extraño lo conocido de manera metafórica a saber: Analogía directa (AD): "Este mecanismo describe la comparación verdadera de hechos paralelos, conocimiento o tecnología."; Analogía personal (AP): “Al identificarse personalmente con los elementos de un problema, el individuo deja de verlo en los términos de los elementos previamente analizados".; Analogía simbólica (AS): “...usa imágenes objetivas e impersonales para describir 
el problema... en términos de respuesta poética." "Es una descripción comprimida de la función o de los elementos del problema..." "...se ve el problema cualitativamente con la súbita totalidad de una frase poética", “....es inmediata. Una vez creada, es un torrente de asociaciones..." y Analogía fantástica (AF): "Como desearíamos que en nuestra fantasía más loca funcionara..." -tal cosa-. Implica la ficción de que "algo va a suceder con sólo desearlo".

En definitiva la analogía antiparadigmática, semeja el sistema donde está el problema en estudio con un sistema ideal, (por ejemplo, comparar el problema de la contaminación que produce un sistema organizacional productivo con el sistema organizacional de las hormigas, para asimilar el procedimiento mediante el cual la organización productiva debería manejar sus residuos sólidos y líquidos con el procedimiento usado por las hormigas para disponer sus residuos y así controlar las fallas del sistema en estudio). Una vez que se ha hecho la analogía antiparadigmática, se procede a generar las ideas de solución de la siguiente manera, Briand, (2004).

\section{Síntesis de ideas de solución}

Comparando las ideas de solución de los actores contrastados y las ideas obtenidas por el consultor mediante la analogía realizada, se organizan todas en una lista a manera de síntesis, sin descartar aquellas que parezcan absurdas (por ejemplo, acabar la organización productiva, seguir con el mismo manejo, cambiar el sentido en el que fluye la materia y la energía en la organización, agregar productos y servicios en la organización que aprovechen el residuo como lo hacen las hormigas con sus desechos, entre otros).

Lo absurdo, ilógico o redundante muestra una faceta de la realidad problema que en últimas no se debe tomar pero que sirve para entender aquello que no se debe hacer y que por lo tanto es parte de la solución, pues el saber que por ahí no se debe ir, es ya una parte del camino para llegar. Según, Ponti, (2006) "a través de la reducción al absurdo nos damos cuenta de obviedades, y sólo a partir de ahí somos capaces de buscar ideas disruptivas", o como lo sugiere Bono, (1967) a partir de la afirmación "los hoteles no tienen habitaciones", en la cual la magia del absurdo reside en saber aprovechar el sinsentido que genera para, así, crear algo nuevo; Bono, (1967) sugiere que "el pensamiento creativo no actúa a través de juicios negativos" conforme a la anterior afirmación "es imposible que un hotel no tenga habitaciones, por tanto es una idea no aprovechable" sino a través de movimientos "un hotel sin habitaciones me hace pensar en un establecimiento que alquile hamacas a un precio muy barato, y te ceda un espacio para instalarla", Bono, (1967).

Esta lista es un conjunto de ideas que busca construir una herramienta importante para el desarrollo de acciones que pueden ser implementadas dentro del sistema solución, con el fin de mejorar la calidad de los procesos y el uso eficiente de los recursos, de tal forma que se pueda mitigar o dar solución integral al problema en estudio.

\section{Planteamiento de la estrategia de solución}

Se basa en la construcción de una matriz de ponderación en la que se establecen los criterios de cumplimiento y de valoración de idea de solución, con el fin de elaborar una estrategia óptima y sistémica, con un orden de prioridad entre cada una de de las ideas de solución planteadas previamente. En la tabla 1 se presenta un ejemplo de matriz de criterios de evaluación y una evaluación de ideas de solución de acuerdo a los criterios establecidos, en ella se encuentra la ponderación de una serie de soluciones para el problema de contaminación de la industria de curtiembres en el municipio de Villapinzon Cundinamarca, y en donde su resultado muestra que la mejor estrategia de solución es el desarrollo del Plan de Manejo Ambiental seguido por una sensibilización y capacitación al personal de la organización. Con esta ponderación se plantea la estrategia de solución para presentársela al dueño del problema.

Los criterios de evaluación podrán ser seleccionados por el consultor según las diversas variables y culturas que se manejen en la 
organización. En el caso mencionado en la tabla 1, se ha escogido los criterios de efectividad (grado en que se logran los objetivos y se resuelven los problemas), eficacia (criterio institucional que revela la capacidad para alcanzar las metas o resultados propuestos), eficiencia (criterio económico que revela la capacidad de producir el máximo de resultados con el mínimo de recursos, energía y tiempo), adaptabilidad (criterio que revela la capacidad de una organización para dar respuesta a un cambio y equilibrarse con este; parte de la adaptabilidad se consigue con la experiencia de la organización), viabilidad (criterio que mide la capacidad de trabajo, funcionamiento y desarrollo adecuado) y proactividad (criterio que mide el actuar con anticipación para hacer frente a una dificultad esperada

\section{Lógica en reversa}

En seguida, se procede en el sistema ITACONE con la evaluación de la estrategia por parte del dueño del problema. El dueño del problema verificará las consecuencias que tiene aplicar la estrategia de solución al problema en estudio, encontrando posibles debilidades, consecuencias negativas en el entorno natural y/o social, posibles limitaciones de presupuesto para implementar la solución, etc. Se denomina este proceso lógica en reversa, porque al utilizarlo se permite replantear un sistema con base en las consecuencias que este tiene donde se aplica, Ballou, (2002): "el proceso de planeación de la cadena de suministros, lleva a cabo y controla el flujo y almacenamiento eficientes y efectivos de bienes y servicios, así como de la información relacionada, desde el punto de origen hasta el punto de consumo, con el fin de satisfacer los requerimientos de los clientes". Ésta etapa del sistema ITACONE permite comprobar la viabilidad de las estrategias de solución, mediante una evaluación detallada entre el consultor y el dueño del problema de dichas ideas con cada uno de los actores identificados dentro del sistema en estudio, con el fin de mantener un equilibrio entre las ideas formuladas originalmente y las propuestas por los actores contrastados.
Hipersolución, es la estrategia de solución definitiva, viable y aplicable al sistema en estudio. La hipersolución se encuentra fundamentada entocnes en las alternativas (ideas de solución) que resultaron del contraste, de la analogía, de la ponderación y de su verificación propuestas entre el consultor y los actores relacionados. Se deberá elaborar un diagrama y un texto que explique la hipersolución como un sistema con sus premisas y puntos de partida, sus recursos necesarios y el cronograma y presupuesto de implementación.

Las soluciones son generadas en consenso proponiendo cambios positivos y sistemas metodológicos aplicables dentro de la organización afectada por el problema a resolver. El consultor deberá cerrar su propuesta de solución mostrando que la hipótesis que tenia para convertir la situación insatisfactoria en ideal es la que lo logra, que aplicar la hipersolución es la mejor alternativa que tiene el dueño del problema y debe poner de presente las consecuencias que tiene aplicar la solución, porque de lo contrario no va a respetar la autonomía del dueño del problema cuando sufra las eventuales consecuencias de la hipersolución propuesta sin ser consciente de ellas; esto determina el carácter bioético del proceder del consultor.

\section{Casos existosos de soluciones creativas obtenidos con itacone}

La búsqueda e implementación de soluciones aceptables para los problemas recurrentes de las organizaciones, ha permitido realizar actividades basadas en la metodología de las soluciones creativas. Estas han sido ejecutadas en las organizaciones foco, dando como resultado un mayor progreso. De esta forma, se presentan en la tabla 2 algunos ejemplos de las soluciones generadas donde se muestra que el uso de la analogía antiparadigmática permitió resolver la diferencia entre la situacion insatisfactoria y la ideal. Finalmente el método Itacone se ha aplicado en organizaciones colombianas, tales como el 
Tabla 2. Casos exitosos en la aplicación de ITACONE para la generación de soluciones creativas a problemas recurrentes.

\begin{tabular}{|c|c|c|c|c|}
\hline $\begin{array}{l}\text { CASO DE } \\
\text { ESTUDIO }\end{array}$ & $\begin{array}{c}\text { SITUACION } \\
\text { INSATISFACTORIA }\end{array}$ & $\begin{array}{l}\text { SITUACION } \\
\text { IDEAL }\end{array}$ & ANALOGIA ANTIPARADIGMÁTICA & ESTRATEGIA DE SOLUCION \\
\hline $\begin{array}{l}\text { Curtiembres } \\
\text { de Villapinzón, } \\
\text { Cundinamarca, } \\
\text { Colombia }\end{array}$ & $\begin{array}{l}\text { Las aguas residuales } \\
\text { se vierten al rio } \\
\text { bogotá y los residuos } \\
\text { sólidos se depositan } \\
\text { en rellenos sanitarios } \\
\text { obsoletos y saturados }\end{array}$ & $\begin{array}{l}\text { minimizar el } \\
\text { vertimiento } \\
\text { de las aguas } \\
\text { residuales y } \\
\text { la generacion } \\
\text { de residuos } \\
\text { solidos con } \\
\text { cromo a } \\
\text { lo rellenos } \\
\text { sanitarios }\end{array}$ & $\begin{array}{c}\text { Ver la curtiembre como una bicicleta } \\
\text { en la que la el cuidado y potencia con } \\
\text { que se hace la fuerza para producir el } \\
\text { movimiento se asimila al cuidado con } \\
\text { el que las personas hacen el tratamiento } \\
\text { de las pieles y en la que las ruedas } \\
\text { de la bicicleta permiten entender que } \\
\text { las tolvas de tratamiento de las pieles } \\
\text { operando eficientemente aumentan el } \\
\text { progreso de la curtiembre sin producir } \\
\text { entropia }\end{array}$ & $\begin{array}{c}\text { Cambiar los insumos de cromo por insumos } \\
\text { biodegradables de tecnologia italiana } \\
\text { Reciclar la sal con el sustituto de cromo para } \\
\text { evitar la putrefaccion del cuero } \\
\text { Realizar raspado de pieles antes de aplicar los } \\
\text { químicos para tratar el cuero } \\
\text { Capacitar a las personas en el manejo del agua } \\
\text { responsable y manejo integrado de residuos } \\
\text { solidos }\end{array}$ \\
\hline $\begin{array}{l}\text { Diseño de una } \\
\text { estrategia de } \\
\text { producción limpia } \\
\text { en la empresa } \\
\text { tintorería el } \\
\text { dorado para el } \\
\text { ahorro y uso } \\
\text { eficiente del agua, } \\
\text { Bogotá, Colombia }\end{array}$ & $\begin{array}{l}\text { Los consumos } \\
\text { mensuales del agua } \\
\text { de la tintorerias } \\
\text { equivalen al } 70 \% \\
\text { del consumo de } \\
\text { las industrias de la } \\
\text { ciudad de Bogotá }\end{array}$ & $\begin{array}{l}\text { Reciclar } \\
\text { el agua sin } \\
\text { producir } \\
\text { efectos } \\
\text { negativos en la } \\
\text { calidad de la } \\
\text { tintura de telas } \\
\text { disminuyendo } \\
\text { el consumo } \\
\text { del agua en la } \\
\text { tintoreria }\end{array}$ & $\begin{array}{c}\text { La asimilacion de la tintoreria a } \\
\text { un Hormiguero permitió ver como } \\
\text { interactuan mejor la planta fisica, los } \\
\text { recursos humanos, las comunicaciones, } \\
\text { las entradas y las salidas del sistema } \\
\text { en relacion con el uso del agua en la } \\
\text { tintoreria y la necesidad de monitorear } \\
\text { su consumo a lo largo de las lineas de } \\
\text { producción }\end{array}$ & $\begin{array}{c}\text { Implementar un sistema de medicion del } \\
\text { consumo del agua al interior de las lineas de } \\
\text { producción en la tintorería } \\
\text { Cambiar los hábitos de consumo de agua y el } \\
\text { uso de los insumos de tinturado a aquellos de } \\
\text { última generación biodegradables } \\
\text { Sustituir el Control del pH en el efluente de } \\
\text { la planta que se hace con Acido Sulfurico } \\
\text { por sustancia acida tipo vinagre para que se } \\
\text { mantenga dentro de los parametros de la norma }\end{array}$ \\
\hline $\begin{array}{l}\text { Plan de gestion } \\
\text { ambiental de } \\
\text { Roseta Industrial, } \\
\text { empresa } \\
\text { que produce } \\
\text { pinturas, Funza, } \\
\text { Cundinamarca, } \\
\text { Colombia }\end{array}$ & $\begin{array}{l}\text { Las aguas residuales } \\
\text { tienen valores de } \\
\text { sólidos suspendidos } \\
\text { de más de } 300 \\
\text { ppm lo que genera } \\
\text { la necesidad de } \\
\text { construir un planta de } \\
\text { tratamiento de aguas } \\
\text { residuales }\end{array}$ & $\begin{array}{l}\text { Verter aguas } \\
\text { residuales que } \\
\text { cumplan con } \\
\text { los parametros } \\
\text { de calidad } \\
\text { del agua de } \\
\text { la norma sin } \\
\text { tener que } \\
\text { hacer planta de } \\
\text { tratamiento }\end{array}$ & $\begin{array}{l}\text { El Baño del cuerpo humano asimilado } \\
\text { a la producción de pinturas de base } \\
\text { acuosa permite ver que el lavado de las } \\
\text { ollas donde se mezcla la pintura se hace } \\
\text { de manera ineficiente, despediciando } \\
\text { aquella que queda untada en los } \\
\text { recipientes, incrementando con ello los } \\
\text { solidos suspendidos en el efluente }\end{array}$ & $\begin{array}{l}\text { Introducir el espatulado en las ollas antes } \\
\text { de lavarlas, lo que evita que la pintura vaya } \\
\text { a la red sanitaria y la cantidad de sólidos } \\
\text { suspendidos sea superior a la norma } \\
\text { Capacitar a las personas en el manejo } \\
\text { responsable del producto pintura durante su } \\
\text { manipulacion, mezclado y envase } \\
\text { Dar a conocer el ciclo del agua a los empleados } \\
\text { y directivos de la planta }\end{array}$ \\
\hline $\begin{array}{c}\text { Plan de ahorro y } \\
\text { uso responsable } \\
\text { del agua en la } \\
\text { Universidad El } \\
\text { Bosque, Bogotá, } \\
\text { Colombia }\end{array}$ & $\begin{array}{l}\text { El consumo del } \\
\text { agua aún durante la } \\
\text { época en la que los } \\
\text { estudiantes no están } \\
\text { en la Universidad } \\
\text { se mantiene un } 20 \% \\
\text { menos que cuando } \\
\text { los estudiantes } \\
\text { y docentes se } \\
\text { encuentran en su } \\
\text { máxima afluencia }\end{array}$ & $\begin{array}{l}\text { El consumo } \\
\text { del agua } \\
\text { debe ser } \\
\text { proporcional } \\
\text { a la presencia } \\
\text { de personal } \\
\text { dentro de la } \\
\text { Universidad }\end{array}$ & $\begin{array}{l}\text { La analogia del uso del agua con la } \\
\text { corriente marina del golfo de México } \\
\text { en el atlántico norte permitió ver y } \\
\text { argumentar eficazmente sobre los } \\
\text { efectos del pobre mantenimiento } \\
\text { de la red hídrica, la necesidad de } \\
\text { implementar un sistema de monitoreo } \\
\text { del lavado de fachadas y pisos con } \\
\text { mejores equipos, la instalacion de } \\
\text { válvulas automáticas en los grifos } \\
\text { de los baños y la implementación } \\
\text { de un sistema de capacitación y } \\
\text { transformación autónoma de la } \\
\text { conducta de estudiantes y docentes para } \\
\text { el uso responsable del agua }\end{array}$ & $\begin{array}{l}\text { Monitoreo del lavado de fachadas y pisos con } \\
\text { equipos de bajo consumo de agua } \\
\text { Instalación de válvulas automáticas en los } \\
\text { grifos de los baños y orinales secos } \\
\text { Reciclaje del agua lluvia e incorporacion de la } \\
\text { misma en la red de aprovisionamiento de los } \\
\text { sanitarios de los edificios de la Universidad } \\
\text { Capacitar continuamente a los estudiantes, } \\
\text { empleados, directivos y docentes con } \\
\text { campañas que los motiven a cambiar sus } \\
\text { hábitos de consumo y su comportamiento } \\
\text { irresponsable con el agua en la Universidad } \\
\text { Implementación de formatos para la } \\
\text { notificación y alerta de fugas hídricas en las } \\
\text { edificaciones de la Universidad }\end{array}$ \\
\hline
\end{tabular}

sistema de gestión ambiental de la Universidad El Bosque, Central de Abastos - Corabastos S.A., en el Resguardo Indígena Arhuaco, en la producción sustentable de alimentos en el Departamento del Meta, Cundinamarca y Huila, así como en varias PYMES Colombianas. La tabla 3, muestra la aplicación del método Itacone dentro de la comunidad indígena arhuaca de la sierra nevada de Santa Marta - Colombia, en donde se diseñó de manera sistémica una organización, que permitiera la interacción armónica entre la medicina tradicional indígena y la medicina occidental; el 
Tabla 3. Caso de la casa de la salud de la ciudad de Nabusímake en la Sierra Nevada de Santa Marta, aplicando el método ITACONE.

\begin{tabular}{|c|c|}
\hline $\begin{array}{l}\text { ETAPA DE APLICACIÓN DE } \\
\text { ITACONE }\end{array}$ & Caso de la Casa de la Salud de la ciudad de Nabusimake en la Sierra Nevada de Santa Marta, Cesar, Colombia. \\
\hline $\begin{array}{l}\text { Documentación de la Percepción } \\
\text { del Problema por el Dueño del } \\
\text { Problema }\end{array}$ & $\begin{array}{l}\text { El Cabildo con el Mamu Kuncha de Nabusimake manifestó la necesidad de organizar y comprender de manera } \\
\text { diferente la casa de la salud que en términos occidentales se conoce como el Centro de Salud y que en ese momento } \\
\text { funcionaba en esa ciudad indígena. }\end{array}$ \\
\hline $\begin{array}{c}\text { Definición y contraste del proble- } \\
\text { ma a resolver }\end{array}$ & $\begin{array}{c}\text { Se hicieron encuestas semiestructuradas a los diferentes líderes indígenas, a los funcionarios del centro de salud, de } \\
\text { miembros usuarios de la comunidad, de autoridades ancestrales, de miembros de la universidad y de funcionarios } \\
\text { del sistema de salud del cesar. El problema resultante fue que a pesar de tener centro de salud en la ciudad, este } \\
\text { debería considerar en su funcionamiento las costumbres y ceremoniales tradicionales para inducir al indígena a su } \\
\text { curación y la prevención de la enfermedad sin traumatismos culturales. }\end{array}$ \\
\hline $\begin{array}{c}\text { Cambio de paradigma mediante } \\
\text { analogía paradigmática }\end{array}$ & $\begin{array}{l}\text { Se hizo la analogía antiparadigmática del funcionamiento de la casa de la salud con el funcionamiento de la célula, } \\
\text { lo cual permitió entender que la función del Mamu en la atención del paciente era la de la membrana celular y que el } \\
\text { paciente era el núcleo de la casa de la salud entre otros, véase la figura } 2 \text {. }\end{array}$ \\
\hline $\begin{array}{l}\text { Generación y ponderación de } \\
\text { ideas de solución }\end{array}$ & $\begin{array}{c}\text { Con una nueva organización matricial que aportara elementos de la cultura en el sentido horizontal y las funciones } \\
\text { comunes de los cargos occidentales en el sentido vertical, se pondero una serie de soluciones que generaron una } \\
\text { enorme satisfacción de quienes se encargaban de promover la salud y prevenir la enfermedad en el Resguardo. Las } \\
\text { ideas de solución consideraron: } \\
\text { Pérdida de identidad por parte del pueblo Arhuaco frente a la cultura occidental: esta situación afecta el libre desa- } \\
\text { rrollo de las costumbres propias ancestrales en temas como salud y alimentación. } \\
\text { Rigidez del presupuesto del Estado colombiano en la comunidad: el cual se encuentra enfocado básicamente a la } \\
\text { detección temprana de enfermedades, promoción y prevención y salud pública, no tiene en cuenta la salvedad de la } \\
\text { ley } 691 \text { en cuanto al pluralismo médico. } \\
\text { Falta unidad de criterio para diseñar un plan de choque frente a las necesidades de la comunidad; no se evidencia un } \\
\text { centro estratégico que realice el proceso gerencial de administrar los recursos (no solo presupuestales) y llevar a cabo } \\
\text { el debido proceso de planeación, organización, dirección y control.Falta de acercamiento entre el personal de salud } \\
\text { occidental y las autoridades de Nabusímake para generar estrategias acordes con los lineamientos biculturales. }\end{array}$ \\
\hline $\begin{array}{l}\text { Verificación de la aplicabilidad } \\
\text { de la estrategia de solución con } \\
\text { logística en reversa }\end{array}$ & $\begin{array}{l}\text { Se conjugaron los aportes de la Universidad con los del Resguardo para generar la aceptación del modelo de la } \\
\text { organización de la casa de la salud en la estructura matricial, produciendo correcciones y mejoramiento de la } \\
\text { estrategia:"el nuevo aporte a la organización de la casa de la salud indígena fue la conformación de un comité de } \\
\text { salud de carácter intercultural, el cual a través de la definición de perfiles decantados en la perspectiva arhuaca, } \\
\text { se provea el humano, el desarrollo de planes interculturales de promoción y prevención, evalué y complemente el } \\
\text { modelo de atención en salud arhuaco, reciba y estudie propuestas de saneamiento ambiental y producción limpia de } \\
\text { la cultura occidental y diseñe planes de educación para la comunidad supervisados por el Mamu" }\end{array}$ \\
\hline Formulación de la hipersolución & $\begin{array}{c}\text { La estructura interna de la organización de la casa la salud se propuso para la IPSI WIntukua, el siguiente modelo } \\
\text { que opera desde las perspectivas financiera, del cliente, de procesos y de aprendizaje y desarrollo que se muestra en } \\
\text { la figura } 2 .\end{array}$ \\
\hline
\end{tabular}

fin de la actividad es brindar atención en servicios de salud de primer nivel a la comunidad Arhuaca en la ciudad de Nabusímake (Sierra Nevada de Santa Marta).

\section{Conclusiones}

El sistema de solución creativa Itacone prepara al consultor para generar soluciones viables, innovadoras y responsables con las organizaciones, produciendo un cambio en el paradigma de las personas que están involucradas en el problema.
Utilizar el sistema Itacone en la solución de los problemas ambientales, ayudará a generar mecanismos eficientes que permitan dar herramientas innovadoras a los problemas que se avecinan y a los que existen actualmente.

Por el uso de Itacone en la obtención de la solución, quien tiene el problema se percata que todas las ideas son valiosas y que la solución efectiva es un sistema de ideas de solución (hipersolución) al que se le prueban los efectos y su aplicabilidad antes de poner en práctica la hipersolución. 

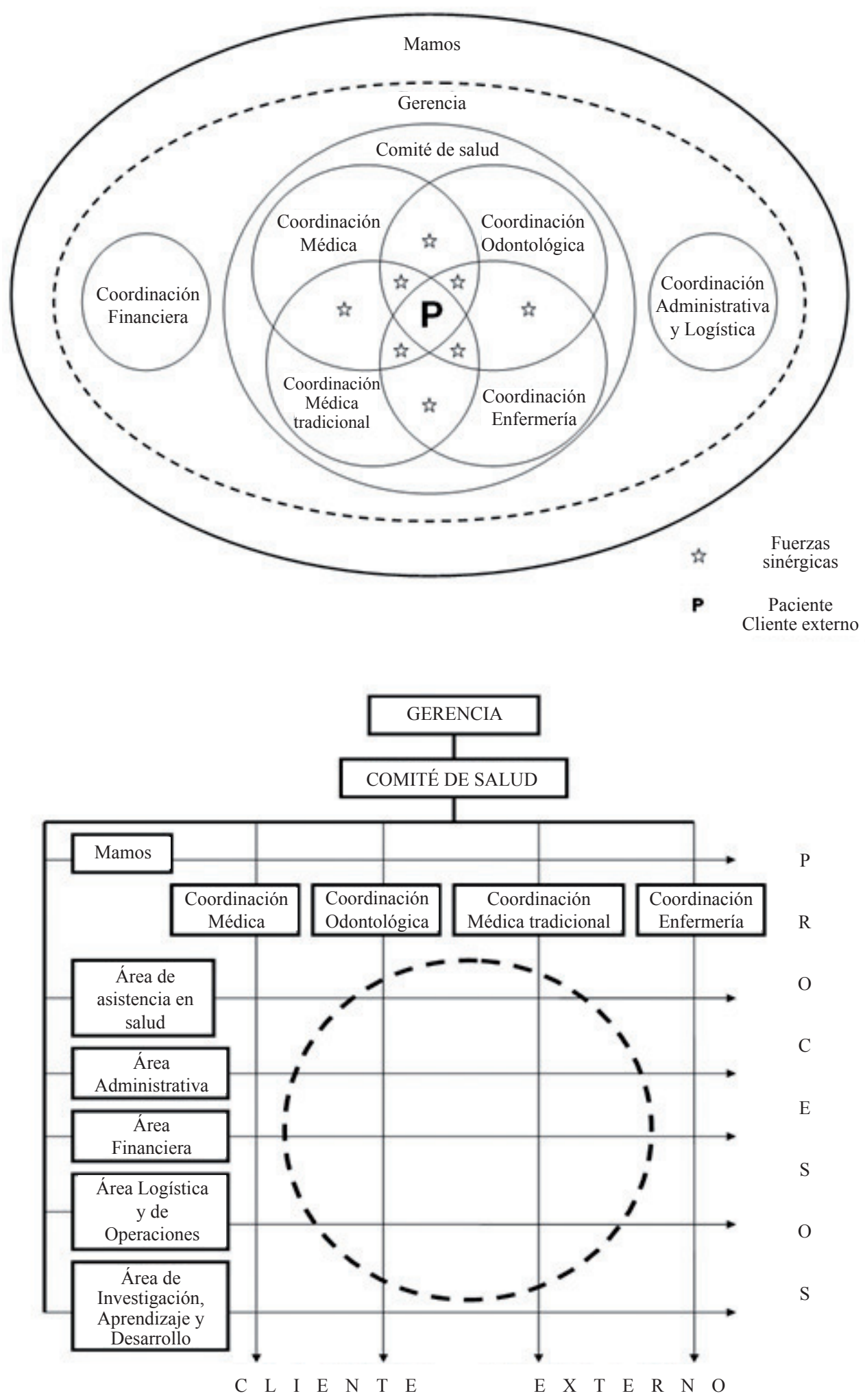

Figura 2. Organigrama célula de la casa del Arhuaco y estructura matricial salud Arhuaca. 


\section{Referencias bibliográficas}

Amabile, T. (2012), Versión revisada en Harvard Business School para Componential Theory of creativity, Working knowledge. http://hbswk.hbs. edu/item/7011.html

Amabile, T. (1997). Motivating Creativity in Organizations: On Doing What You Love and Loving What You Do, California Management, Review, 40(1), 39-58.

Amabile T.M, (1988). A Model of Creativity and Innovation in Organizations en B. M. Staw y L.L. Cummings (eds), Research in Organizational Behavior, 10, 126.

Angulo, J. (2000). Versión revisada de todo logistica sobre Logística Inversa. http://www. todologistica.com/online/site/58692I1.php.

Alexander, T. (1992). "Sinéctica: la invención por el método de la locura". en Gary a.Davis y J.A. Scott (comps), Estrategias para la Creatividad, 68. Buenos Aires :Ed. Paidos Educador.

Almiron, A. (2010). Técnicas para el desarrollo de la creatividad, Consultado el 21 Junio 2012 en: http://alejandralmiron.fullblog.com.ar/tecnicasdesarrollo-de-la-creatividad.html

Ballou, R. (2002). Logística Administración de la cadena de suministro. México: Pearson Educación.

Bono, E. (1967). New Think: The Use of Lateral Thinking. New York: Basic Books.

Briand, A (2004). Proceso de Examinación Creativa PEC. Canadá: Université du Quebec à Chocoutimi.

Brown, L.R. (2009). Plan B 3.0 Movilizarse para salvar la civilización. Bogotá: Kimpres Ltda.

Esquivas, M. (2004). Creatividad: Definiciones, antecedentes y aportaciones. Revista Digital Universitaria, DGSCA-UNAM 5 (1), 1-17
Fabris, F., Lachowicz G. \& Puccini S. (2008). Versión revisada de la espiral dialectica para la Sinéctica, aplicación de una técnica creativa. http://www.espiraldialectica.com.ar/sinectic.htm

Feist, G. \& Barron, F. (2003). Predicting Creativity from Early to late Adulthood Intellect, Potential and Personality. Journal of Research in Personality, 37 (2), 62-88.

Gary, D. \& Scott, J. (2007). Estrategias para la Creatividad. Buenos Aires: Paidos Educador.

Gary, S. (2007), 12500 frases célebres, Grupo Editorial Tomo SA de CV.

Godoy, G. y Romero J. (2005). Diagnóstico y propuesta de mejoramiento limpio para la industria de curtiembres en el municipio de Villapinzon- Cundinamarca. Trabajo de grado. Universidad el Bosque, Bogotá D.C, Colombia.

Gordon, W. (1961), Synectics. Nueva York : Harper \& Row.

Gordon, W. (1963). Sinéctica. El desarrollo de la capacidad creadora, Mexico. Herero Hermanos Sucesores.

Gordon, W. (1992). "Sinéctica: historia, evolución y métodos", en Gary, D \& Scott, J. (2007), Estrategias para la Creatividad. Buenos Aires. Paidos Educador.

Guzmán J., Romero J. A. (2008). Diseño Organizacional de un hospital de primer nivel para el pueblo Wintukwa en la ciudad de Nabusimake - Sierra Nevada de Santa Marta. Trabajo de Grado. Universidad El Bosque, Facultad de Ciencias Económicas y Administrativas, Bogotá, Colombia.

Maya, A. (1995), Desarrollo sostenible: aproximaciones conceptuales. Quito: UICN Fundación Natura.

Moccio, F. \& Amábile, B. (1991). El valor terapéutico del entrenamiento en creatividad. Revista Temas de Psicología, 5 (12), 30-45. 
Mora, M. (2006). Metodología de la Investigación. Desarrollo de la Inteligencia. México. Thomson.

Perry-Smith E. y C.E. (2003). The social Side of Creativity: A Static and Dynamic Social Network Perspective, Academy of Management Review, 28, 89-106.

Ponti, F. (2006). Siete estrategias de creatividad. Gestión, Organización y Dirección de R.R.H.H. , En VI seminario conectividad para la educación y la sociedad innovación. Pereira, Colombia, p. 80 - 96.
Robbins, S. and Judge, T. (2009), Comportamiento Organizacional, En: Pearson/Prentice Hall. (Capitulo 5).

Triana, M. (1972). La Civilización Chibcha. Colombia: Escuela Topográfica Saleciana.

Woodman, W. J. E. Sawyer \& Griffin, R. (1993). Toward a Theory of Organizational Creativity. Academy of Management Review, 18 (2), 298. 